\title{
A Synopsis on COVID-19 and Associated Risk Factors: Optimizing Preventive and Clinical Outcomes through Lifestyle Intervention
}

\author{
Abiodun Bamidele Adelowo \\ Department of Public health, Texila American University, Guyana
}

\begin{abstract}
Since its outbreak in late 2019, Coronavirus Disease 2019 (COVID-19) has been ravaging the health system of most countries of the world. Although many preventive and treatment strategies have been proposed and implemented to combat the disease, these efforts seem to be insufficient, and in some cases ineffective. This is evident by the daily rising global incidence and case fatality of the COVID-19 pandemic. A situation if not mitigated early will likely crumble the global economy and tilt the world to an unprecedented global recession. This challenge demands that researchers and clinicians ask more in-depth questions about the novel coronavirus disease. Aside from age that has been confirmed to be linearly associated, what are the other possible socio-demographic and lifestyle-related risk factors that may be associated with COVID-19? What are the possible factors or comorbid conditions that may worsen clinical progression and determine the clinical outcome in confirmed COVID-19 cases? Does the pre-, peri-, or post-morbid lifestyle choices of people have an impact on COVID-19 preventive and treatment efforts? And how can we use the knowledge of the associated risk factors, comorbid conditions, and lifestyle choices of people to improve preventive efforts and clinical management of COVID-19? Answer to these questions may likely serve as an important guide for policymakers and clinicians in their design and implementation of COVID-19 targeted preventive and treatment policies and programs, especially in Low- and Middle-Income Countries (LMICs). The purpose of this article is to critically review available literature and provide evidence-based recommendations.
\end{abstract}

Keywords: COVID-19 pandemic, lifestyle-related COVID-19 risk factors, chronic non-communicable diseases, COVID-19 prevention, COVID-19 clinical outcomes, lifestyle intervention.

\section{Introduction}

Coronavirus Disease 2019 (COVID-19) was first reported in Wuhan City, Hubei Province, China in December 2019, and within a few months, it has spread rapidly to most countries of the world. This prompted the World Health Organization (WHO) to declare it a Public Health Emergency of International Concern (PHEIC), and by 12 March 2020, it was declared a global pandemic [1, 2, 3, 4]. By this declaration, COVID-19 became one of the few diseases to attain a global pandemic status in recent human history. As of 28 January 2021, COVID-19 has been confirmed in 100,455,529 people, with associated 2,166,440 deaths worldwide [5]. The striking characteristics that drew so much attention to COVID-19 are its high infectivity and fatality rates among the general public and the severely ill patients, respectively.
Globally, the care fatality rate (CFR) of COVID-19 has been noticed to rise rapidly with increasing age; the CFR is $<1 \%$ for patients $<50$ years of age, rising to $1.3 \%$ for 50 -year-old patients, to $3.6 \%$ for 60 -year-old patients, to $8 \%$ for septuagenarians, and $14.8 \%$ for octogenarians [6]. Unfortunately, according to a recent interim report by the WHO Solidarity trial consortium, the commonly used COVID-19 treatment is regimens-Remdesivir, Hydroxychloroquine, Lopinavir, and Interferonappeared to have little or no significant effect on the hospitalization and CFR [7]. For these reasons, this article intends to deepen the conversation on the relationship between the clinical progression of COVID-19 and some related risk factors, and how such knowledge can be harnessed to improve preventive and treatment efforts. 


\section{Methods}

An extensive search (with no language restrictions) was done in Google search engine, Academia, ResearchGate, and MEDLINE database. The websites of reputable public health organizations, like the WHO, United Nations, United Nations Children's Fund (UNICEF), Public Health Ontario, Centre for Disease Control (CDC), and Nigeria Centre for Disease Control (NCDC) were also visited for relevant and current information. Some of the keywords that were searched include COVID19, COVID-19 risk factors, clinical outcome in COVID-19, underlying comorbid diseases in COVID-19, etc. All the obtained data were collected, visualized, profiled, cleansed, prepared, analyzed, and summarized accordingly.

\section{Socio-Demographic Factors and COVID- 19}

Although the entire disease evolution of COVID-19 is not yet fully understood, for now, it appears the disease has a preference for certain socio-demography in the society. Multiple systematic reviews of COVID-19 cases have suggested that the infectivity, hospitalization, and fatality rates are more in the people who are 60 years and above, male gender, and ethnic minority groups. In particular, people of the black race seem to have three times the infectivity rate, and almost six times the rate of deaths, compared to the Caucasians [8]. A retrospective observational study that was done among 1, 591 COVID-19 patients in Italy collaborated these observations. The study discovered that out of all the confirmed COVID19 cases, the infected male population was $82 \%$, the females were $18 \%$, while the median age was 63 years [9]. Particularly among the age groups of 51-60, 61-70, and 71-80 years, the majority of patients were males $(83 \%, 81 \%$, and $82 \%$, respectively) compared to the females (17\%, $19 \%$, and $18 \%$, respectively) [9]. Other studies from China revealed that almost $50 \%$ of patients that were diagnosed with "refractory" COVID19 were of the male gender, older age, and with underlying comorbidities $[9,10]$.

Another study on 41 COVID-19 patients on admission in China, revealed that majority of the infected patients were men (73\%), with a median age of 49.0 years [9]. Yet, another study in China, that involved 69 COVID-19 patients, revealed that $68 \%$ were male, with a mean age of 55.5 years [9]. A retrospective study also in China, with 138 hospitalized patients with confirmed COVID-19, shows that $54.3 \%$ were men, while their median age of 56 years [9]. Also, another study that was done among 1,099 patients with COVID-19 from 552 hospitals in 30 provinces in China, revealed a median age of 47 years, and that $58 \%$ of the patients were of the male gender [9]. However, one study in China comprising 140 confirmed COVID-19 patients, reported a different result. It found an equal distribution by sex, at approximately $1: 1$ ratio of male $(50.7 \%)$ and female $(49.3 \%)$, while majority $(70 \%)$ of them were over 50 years old [9]. A study that investigated 5,700 hospitalized COVID-19 patients in 12 New York City area hospitals, also noticed that the median age of the patient was 63 years, out of which $60.3 \%$ and $51.5 \%$ were males and African-American (and other minority ethnic groups), respectively [11].

\section{Pre-Morbid Lifestyle-Related Risk Factors and COVID-19}

Everybody regardless of their health status can be infected with SARS-CoV-2 [12, 13]. However, evidence abounds that suggest that certain lifestyle-related factors or practices tend to predispose some group of people to COVID19 compare to others. Some of the identified predisposing factors include smoking, overweight/obesity, and hypovitaminosis D. Hypovitaminosis D may result from either poor nutrition/unhealthy diet or reduced exposure to sunlight due to a sedentary lifestyle $[14,15]$.

Studies on tobacco smoking in particular have generated different contrasting results. A study that investigated the relationship between COVID-19 and smoking observed that the percentage of current smoker among COVID-19 patients was low and could not explain the association between smoking and susceptibility to SARS-CoV-2 [9]. One early study even suggested that smoking may be protective against SARS-CoV-2 [16]. However, multiple studies and positional statements by the WHO and other international public health organisations have subsequently clearly associated smoking with a higher probability of contracting SARS-CoV-2 and developing a severe form of COVID-19 [17, 18]. Tobacco smoking tends to evoke adverse pathophysiological changes in the respiratory 
and other systems of the smokers, which tend to worsen the clinical progression of COVID-19. A situation that may progress to ICU admission, ventilator support, or death from related complications [19]. A study on Chinese with confirmed COVID-19 with a history of smoking noticed that the patients had a $14 \%$ higher risk of developing COVID-19 induced pneumonia, and 14 times higher Odd Ratio (OR) of progressing to stage III (severe stage) or dying from COVID19 complications [20]. Some studies have even observed significantly higher ACE2 (the host receptor for SARS-CoV-2) gene expression in former and current smoker's lungs compared to non-smoker's lungs $[9,17]$.

Hypovitaminosis D is another risk factor that has been associated with increase susceptibility and severity of COVID-19 [9, 21]. In a study that was conducted in Spain on 216 hospitalized COVID-19 patients, it was discovered that $82.2 \%$ of the patients were vitamin D deficient, compared to $47.2 \%$ of healthy people of same gender and age [22]. Another study that was conducted in Italy on hospitalized COVID-19 patients, noticed that about $50 \%$ of patients with severe vitamin $\mathrm{D}$ deficiency progressed to a severe form of COVID-19 and died, compared to only 5\% of COVID-19 related death in patients with normal serum level of Vitamin D [22]. Other studies have demonstrated that higher serum level of vitamin D correlate with milder clinical presentation of COVID-19, while countries with high serum levels of vitamin D tend to have lower COVID-19 related case fatality rates [22].

It is also worth noting that since studies have shown a positive correlation between decreasing serum vitamin D levels and increasing age, male gender, and obesity [9, 23], this may be some of the reason why COVID-19 is more fatal in these group of people. Low serum Vitamin D may also be related to the regular consumption of certain unhealthy diets that are low in vegetables and fruits $[14,15]$. A sedentary lifestyle may also indirectly lead to reduce serum level of Vitamin D. Here, the affected individuals could not get adequate exposure to sunlight due to inadequate outdoor physical activity [24]. Consequently, vitamin D rich diets and supplements, with adequate exposure to sunlight, would likely be valid lifestyle intervention measures for preventing and treating COVID-19 among atrisk and confirmed cases, respectively $[9,25]$.

\section{Lifestyle-Related Co-Morbid Conditions and COVID-19}

Multiple studies have indicated that people with certain lifestyle-related underlying disease conditions are more susceptible to contracting SARS-CoV-2, compared to people that are free of these diseases. Aside from age, sex, ethnicity, and lifestyle-related risk factors, other factors that may increase the susceptibility of people to contracting SARS-CoV-2 and developing a severe form of COVID-19, includes underlying lifestyle-related chronic medical conditions, such as chronic obstructive pulmonary diseases (COPD), cardiovascular diseases (like heart disease and stroke), high blood pressure/hypertension, diabetes mellitus, kidney disease, liver disease, some cancers (like leukemia, lymphoma or myeloma) $[12,13,26$, 27].

COVID-19 patients with comorbid diabetes mellitus in particular usually have a significantly increased risk of hospitalization and death compared to other underlying disease conditions $[28,29]$. It is not yet fully understood why diabetes mellitus predisposes to higher susceptibility and severity of COVID-19, but it is well known that diabetic patients usually develop a dysregulated immune system and are generally more susceptible to infections, compared to non-diabetic cases $[9,28]$. Also, most infective processes (viral or otherwise) generally tend to progress faster and worse in diabetes (especially if poorly controlled) compared to non-diabetic cases [30]. Moreover, the late clinical stages of type 2 diabetes have been associated with a distorted functionality of ACE2 receptors, the host receptors for SARSCoV-2 [9].

Available studies also collaborated with the above position on the association of COVID-19 with some comorbid conditions. A meta-analysis involving seven studies and 1, 576 COVID-19 patients, revealed that a significant number of the patients had an underlying chronic disease condition. The most prevalent comorbidities were hypertension $(21.1 \%)$ and diabetes mellitus $(9.7 \%)$, followed by cardiovascular disease $(8.4 \%)$ and respiratory system disease $(1.5 \%)$ [31]. Another study was conducted on 7,736 confirmed COVID-19 patients in Wuhan China, out of which $23.7 \%$ had at least one coexisting chronic disease [32]. In a study conducted in Italy to investigate the association of COVID-19 
with lifestyle-related chronic diseases, hypertension was the most common comorbidity (49\%), followed by cardiovascular disease (21\%), hypercholesterolemia (18\%), and diabetes mellitus (17\%) [9]. A meta-analysis that was conducted in China revealed that the following characteristics are common among deceased COVID-19 cases-advanced age ( $>60$ years), cancers, other underlying chronic diseases, and/or major secondary infections [9]. Another China-based retrospective study on 799 COVID-19 in-patients recorded a $14.1 \%$ mortality rate. The study noticed that certain underlying chronic diseases, like hypertension (48\%) and other CVDs (14\%) were more frequent among deceased patients (24\%) compared to the recovered patients (4\%) [9].

The report of a study of hospitalized COVID19 cases still in China, showed that out of the $26.1 \%$ of patients that were transferred to ICU, most $(72.2 \%)$ had concurrent comorbidities, as opposed to only $37.3 \%$ of patients who did not require ICU care [33]. Among the 1, 099 confirmed COVID-19 cases that were reported in a study, 173 had severe disease presentations. Compared with the non-severe cases, the patients with the severe disease had a higher prevalence of hypertension $(23.75 \%$ versus $13.4 \%)$, diabetes mellitus ( $16.2 \%$ versus $5.7 \%)$, coronary heart disease $(5.8 \%$ versus $1.8 \%)$, COPD $(3.5 \%$ versus $0.6 \%)$ and cerebrovascular disease (2.3\% versus $1.2 \%)$ [33]. A recently conducted meta-analysis also concluded that patients with hypertension, respiratory disease, and CVD had an odd ratio of 2.36, 2.46, and 3.42 , respectively, for developing severe COVID-19 compared with the non-severe cases [33]. Other lifestyle-related underlying chronic diseases that studies have associated with a severe form of COVID-19 are chronic liver disease (CLD) and chronic kidney disease (CKD) $[34,35]$. In most cases, deaths of COVID-19 patients did not primarily result from the disease condition itself [27]. Rather, it resulted from complications arising from the underlying chronic conditions, which in most cases became worse as a result of the acute inflammatory assault of COVID-19 on the multiple organs of the body [27].

\section{Optimizing Preventive and Treatment Efforts in COVID Management through Lifestyle Intervention}

The review strongly suggests that although COVID-19 is primarily a respiratory disease, it is associated with some risk factors that either predispose people to the disease or worsen its clinical outcome. The three main sociodemographic risk factors that are implicated in COVID-19-older age group ( $\geq 60$ years), male gender, and black race-are all well documented non-modifiable risk factors for chronic noncommunicable diseases (NCDs) like hypertension, cardiovascular disease, type 2 diabetes mellitus, and some cancers [36, 37]. The directly implicated lifestyle-related risk factors for contracting SARS-CoV-2 or developing a severe form of COVID-19 are the history of smoking/current smoking, overweight/obesity, high blood pressure, hypercholesterolemia, and hypovitaminosis D. Indirectly, risk factors like poor nutrition/unhealthy diet and outdoor physical inactivity (with poor exposure to sunlight), are also implicated since such habits may likely lead to hypovitaminosis D. These direct and indirect risk factors are also well-documented modifiable risk factors for most chronic NCDs [38]. Chronic NCDs-like chronic obstructive pulmonary diseases (COPD), hypertension, cardiovascular diseases (heart disease, stroke), diabetes mellitus, chronic kidney disease, chronic liver disease, and some cancers (like leukemia, lymphoma or myeloma) were also identified as underlying co-morbid conditions that are associated with and modifies clinical progression and outcomes in COVID-19. The presence of these underlying chronic diseases in many cases tend to increase COVID-19 susceptibility, progression, and worsen the clinical outcome.

Many pieces of evidence have also strongly associated these chronic co-morbid diseases with unhealthy lifestyle choices like unhealthy diets, tobacco use, and physical inactivity [38]. Overweight/obesity, high blood pressure, and hypercholesterolemia, are also lifestyle-related intermediate risk factors for these chronic 
co-morbidities [36]. According to the WHO and some other scientists, some healthy lifestyle choices, if practiced consistently and correctly, have been clinically proven to be effective in controlling these COVID-19 associated modifiable risk factors, and ultimately prevent, control, or even reverse some of the underlying chronic diseases [39, 40]. Some of the well documented healthy lifestyle choices that are capable of performing these tasks include healthy diet, adequate physical activity (especially outdoor), adequate restorative sleep, good stress management, avoidance of alcohol abuse, good mental and psychological health, and social connectivity [39, 40, 41]. During the pre-COVID-19 state, the practice of healthy lifestyle as a preventive effort in addition to other preventive measures will likely reduce the infectivity rate of SARS-CoV-2 [25] and flatten the COVID-19 pandemic curve faster (see Figure 1) [42].

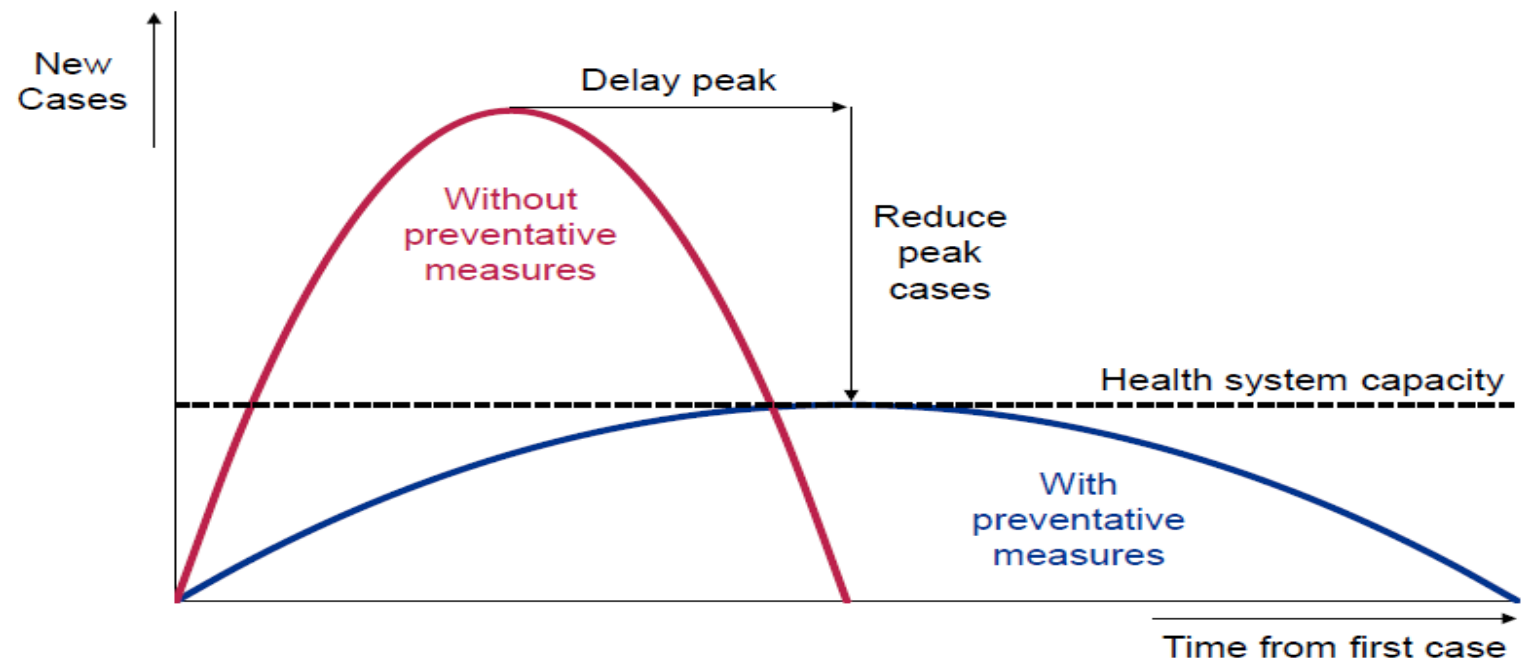

Figure 1. Potential Effect of Preventive Measures on the COVID-19 Pandemic Curve

As depicted by the graph, preventive measures (that include measures like hand washing, physical distancing, healthy lifestyle, etc.), are vital in the global efforts to quickly flatten the COVID-19 pandemic.

For the peri-COVID-19 (confirmed positive COVID-19) state, lifestyle modification will likely slow down the disease progression, stabilize underlying chronic diseases, and significantly improve clinical outcomes, with a consequential reduction in case fatality rate [43]. This lifestyle interventions might be particularly important in cases with poor response to pharmacological intervention. During postCOVID-19 (confirmed negative COVID-19) state, consistent healthy lifestyle practice will likely ensure early recovery of the assaulted multiple organs, stabilizes the underlying disease conditions, and ensure the patients quickly regain their pre-morbid functionality with faster reintegration to the larger society. Some of the general lifestyle intervention principles that should be considered in the management of pre-, peri-, and/or post-COVID19 cases can be found in Table 1 [40, 44, 45, 46].

Table 1. General Lifestyle Intervention Recommendations in COVID-19 Management

\begin{tabular}{|l|l|}
\hline Categories & Recommendations \\
\hline \multirow{3}{*}{ Nutrition } & $\begin{array}{l}\text { Make healthy food choices. The food should be low in saturated fats, trans-fat and } \\
\text { calories, but high in fiber and other nutrients. Such should contain a significant } \\
\text { number of fruits, vegetables, whole grains, legumes, nuts, and seeds. }\end{array}$ \\
\cline { 2 - 3 } Physical Activity & $\begin{array}{l}\text { De physically active. Engage in moderate-intensity aerobic physical activity } \\
\text { (preferably outdoor) of } 30 \text { minutes to } 1 \text { hour every day. This should be done } \\
\text { without compromising the physical distancing rules. }\end{array}$ \\
\hline $\begin{array}{l}\text { Weight } \\
\text { Management }\end{array}$ & $\begin{array}{l}\text { Maintain body mass index (BMI) between } 18.5 \text { to } 24.9 \mathrm{kgm}^{-2} \text { and waist-hip ratio } \\
\text { to less than } 0.8 \text { in women and } 0.9 \text { in men. }\end{array}$ \\
\hline
\end{tabular}




\begin{tabular}{|l|l|}
\hline \multirow{2}{*}{$\begin{array}{l}\text { Harmful } \\
\text { Substances }\end{array}$} & Quit tobacco use, reduce the amount smoked, or do not start the habit. \\
\cline { 2 - 3 } Sleep & If you must drink alcohol, do so in moderation and it should not be every day. \\
\hline Stress & $\begin{array}{l}\text { Adopt a variety of measures to keep stress levels low all the time. Such measures } \\
\text { may include adequate planning of daily activity, adequate coping skills, deep } \\
\text { breathing, meditation, mindfulness, and Yoga. }\end{array}$ \\
\hline $\begin{array}{l}\text { Psychological } \\
\text { Wellbeing }\end{array}$ & $\begin{array}{l}\text { Ensure positive psychological and mental wellbeing all the time with a positive } \\
\text { outlook of situations. Ensure social connection with loved ones, without } \\
\text { compromising the physical distancing rules. }\end{array}$ \\
\hline
\end{tabular}

\section{Conclusion}

Policymakers should carefully consider the association between COVID-19 and some sociodemographic risk factors, lifestyle-related risk factors, and underlying chronic disease. A comprehensive COVID-19 prevention guideline, that emphasizes healthy lifestyle, should be designed to target the general population, but most importantly at-risk population (people whose socio-demography, lifestyle, or occupational status places them at higher risk of contracting SARS-CoV-2).

\section{References}

[1] Beeching N.J., Fletcher T.E., and Fowler R., 2020, BMJ Best Practice: Coronavirus disease 2019 (COVID-19 leaflet), BMJ Publishing Group Ltd 2020, Date of access: 4/7/2020. http://patchsa.org/wpcontent/uploads/2020/03/BMJ-Best-PracticeCOVID-19.pdf.

[2] Bonyan F.A., Shareef L.G., Al-waily A., Abdulrazaq A.A., and Al-Rubayee W.A., 2020, COVID-19 clinical characteristics and outcomes in 60 hospitalized Iraqi patients - Case series. Medical Science, 24(104), 2251-2258.

[3] Chowdhury A.T.M.M, Karim M. R., Mehedi H.M.H, Shahbaz M., Chowdhury M.W., Dan G., et al., 2020, Analysis of the primary presenting symptoms and hematological findings of COVID19 patients in Bangladesh, ResearchGate 2020. DOI: 10.13140/RG.2.2.22406.80969.

[4] Nigeria Centre for Disease Control, 2020, National interim guidelines for clinical management of COVID-19, NCDC 2020, Date of access: $14 / 10 / 2020$.

https://reliefweb.int/sites/reliefweb.int/files/resource s/national_covid19_case_management_guide_ncdc. pdf.
Clinical management guidelines for the confirmed COVID-19 cases should also integrate comprehensive lifestyle modification in the management of cases regardless of the disease stage. Healthy lifestyle should also be incorporated in the rehabilitative phase of confirmed COVID-19 negative patients. Such measures will likely reduce significantly the incidence and case fatality rates of COVID-19 and also facilitate the early restoration of functionality of patients that were severely affected by COVID- 19 .

[5] World Health Organisation, 2020, WHO Coronavirus Disease (COVID-19) Dashboard, Date of access: 14/10/2020. https://covid19.who.int/.

[6] Clerkin K.J., Fried J.A., Raikhelkar J., Sayer G., Griffin J.M., Masoumi A., et al., 2020, COVID-19 and Cardiovascular Disease, Circulation, 141: 1648 1655 ,

DOI: 10.1161/CIRCULATIONAHA.120.046941.

[7] Pan H., Peto R., Karim Q.A., Alejandria M., Henao-Restrepo A.M., García C.H., et al., 2020, Repurposed antiviral drugs for COVID-19-interim WHO SOLIDARITY trial results: WHO Solidarity trial consortium, WHO 2020, Date of access: 18/10/2020.

https://doi.org/10.1101/2020.10.15.20209817.

[8] Khunti K., Singh A.K., Pareek M., and Hanif W., 2020, Is ethnicity linked to incidence or outcomes of covid-19? Preliminary signals must be explored urgently, BMJ, 369: 1 - 2. DOI: 10.1136/bmj.m1548. [9] Ambrosino I., Barbagelata E., Ortona E., Ruggieri A., Massiah G., Giannico O.V., et al., 2020, Gender differences in patients with COVID-19: a narrative review, Monaldi Archives for Chest Disease, 90 (1389): $318-324$.

[10] Mo P., Xing Y., Xiao Y., Deng L., Zhao Q., Wang H., et al., 2020. Characteristics of refractory 
COVID-19 pneumonia in Wuhan, China. Infectious Diseases Society of America, 2020, Oxford University Press, Date of access: 9/10/2020. https://watermark.silverchair.com/ciaa270.pdf?toke $\mathrm{n}=$.

[11] Richardson S., Hirsch J.S., Narasimhan M., Crawford J.M., McGinn T., and Davidson K.W., 2020, Presenting Characteristics, Comorbidities, and Outcomes Among 5, 700 Patients Hospitalized With COVID-19 in the New York City Area, JAMA, 323(20): $2052 \quad-\quad 2059$. DOI:10.1001/jama.2020.6775.

[12] Public Health Ontario, 2020, Coronavirus Disease 2019 (COVID-19): How to Protect Yourself from COVID-19 - Older Adults and People with Chronic Medical Conditions or Weakened Immune Systems. Queen's Printer for Ontario 2020, Date of access:

14/10/2020.

https://www.publichealthontario.ca/-

/media/documents/ncov/factsheet/2020/05/factsheet -covid-19-immunocompromised.pdf?la=en.

[13] National Association of Chronic Disease Directors, 2020, Chronic Disease and COVID-19: What You Need to Know Tips and information to protect yourself and your family, National Association of Chronic Disease Directors 2020.

Date of access: 15/10/2020. https://cdn.ymaws.com/www.chronicdisease.org/re source/resmgr/website-

2020/covid/factsheet_covid19cd.pdf.

[14] Bener A., Alsaied A., Al-Ali M., Hassan A.S., Basha B., Al-Kubaisi A., et al., 2009, Impact of Lifestyle and Dietary Habits on Hypovitaminosis D in Type 1 Diabetes Mellitus and Healthy Children from Qatar, a Sun-Rich Country, Annals of Nutrition \& Metabolism, 53 (3/4): 215 - 222. https://www.jstor.org/stable/48507937.

[15]Zgaga L., Theodoratou E., Farrington S.M., Agakov F., Tenesa A., Walker M., et al., 2011, Diet, Environmental Factors, and Lifestyle Underlie the High Prevalence of Vitamin D Deficiency in Healthy Adults in Scotland, and Supplementation Reduces the Proportion That Are Severely Deficient, The Journal of Nutrition, 6: 1535 - 42. doi:10.3945/jn.111.140012.

[16] Bonevski B., 2020. Does nicotine protect us against coronavirus? The Conversation Africa, Inc. 2020. Date of access: 10/09/2020. https://theconversation.com/does-nicotineprotectus-against-coronavirus-137488.

[17] van Zyl-Smit R., Richards G., and Leone F.T., 2020, Tobacco smoking and COVID-19 infection, thelancet.com/respiratory, 8: $664-5$.
[18] World Health Organization, 2020, WHO statement: Tobacco use and COVID-19. World Health Organization 2020. Date of access: 14/10/2020.

https://www.who.int/newsroom/detail/11-05-2020who-statement-tobacco-use-and-covid- 19.

[19] Banerjee B, and Banerjee R., 2020, Role of tobacco in SARS-CoV-2 infection and COVID-19: A scoping review. Int J Non-Commun Dis, 5:70 - 5. [20] Yu G., 2020, How smoking, vaping and drug use might increase risks from Covid-19. CNN 2020. Date of access: 18/10/2020. https://edition.cnn.com/2020/03/20/health/coronavi rus-vaping-drugs/index.html.

[21] Landsverk G., 2020, There's new evidence vitamin D may help treat COVID-19 by reducing the severity of infection. Insider Inc 2020. Date of access: 28/10/2020. https://www.insider.com/newevidence-suggests-vitamin-d-could-reducecoronavirus-severity-2020-10.

[22] Miller A.M., 2020, Over $80 \%$ of coronavirus patients had vitamin $D$ deficiency in one Spanish hospital. Insider Inc 2020. Date of access: 28/10/2020. https://www.insider.com/80-percentof-hospitalized-coronavirus-patients-had-vitamind-deficiency-2020-10.

[23] Nasri H., and Ardalan M.R., 2012, Association of serum vitamin D level with age in individuals with normal renal function, J Nephropharmacol, 1(1): 7-9.

[24] Roomi M.A., Farooq A., Ullah E., and Lone K.P., 2015, Hypovitaminosis D and its association with lifestyle factors, Pak J Med Sci, 31(5): 1236 1240. http://dx.doi.org/10.12669/pjms.315.7196.

[25] World Health Organization, 2020, Nutrition advice for adults during the COVID-19 outbreak. World Health Organization 2020. Date of access: 27/08/2020.

http://www.emro.who.int/images/stories/nutrition/d ocuments/en_flyer_nutrition_adults_covid_19.pdf? ua $=1$.

[26] World Health Organization, 2020, Clinical management of COVID-19: Interim guidance. WHO 2020, Date of access: 15/10/2020? https://www.who.int/publications/i/item/clinicalmanagement-of-covid-19.

[27] Wang L., 2020, C-reactive protein levels in the early stage of COVID-19, Médecine et maladies infectieuses, 50: 332-334.

[28] Apicella M., Campopiano M.C., Mantuano M., Mazoni L., Coppelli A., and Del Prato S., 2020, COVID-19 in people with diabetes: understanding 
the reasons for worse outcomes. thelancet.com/diabetes-endocrinology, 8: $782-92$. [29] Pugliese G., Vitale M., Resi V., and Orsi E., 2020, Is diabetes mellitus a risk factor for Coronavirus Disease 19 (COVID-19)? Acta Diabetol., 31: 1 - 11. doi: 10.1007/s00592-02001586-6.

[30] Casqueiro J., Casqueiro J., and Alves C., 2012, Infections in patients with diabetes mellitus: A review of pathogenesis, Indian Journal of Endocrinology and Metabolism, 16 (1): 27 - 36. DOI: $10.4103 / 2230-8210.94253$.

[31] Yang J., Zheng Y., Gou X., Pu K., Chen Z., Guo Q., et al., 2020, Prevalence of comorbidities and its effects in patients infected with SARS-CoV-2: a systematic review and meta-analysis. International Journal of Infectious Diseases, 94: 91-95. https://doi.org/10.1016/j.ijid.2020.03.017.

[32] Guan W., Ni Z., Hu Y., Liang W., Ou C., He J. et al., 2020, Clinical Characteristics of Coronavirus Disease 2019 in China, $n$ engl $j$ med, 382: 1708 1720, DOI: 10.1056/NEJMoa2002032.

[33] Pal R., and Bhadada S.K., 2020, COVID-19 and non-communicable diseases, Postgrad Med J, 96: $429-430$

[34] Verma N., Duseja A., and Singh V., 2020, Impact of Pre-existing Chronic Liver Disease on the Outcome of Patients with COVID-19 Disease, Gastroenterology 2020, doi: https://doi.org/10.1053/j.gastro.2020.05.090. Date of access: 15/10/2020. https://www.gastrojournal.org/article/S00165085(20)34782-X/pdf.

[35] Oyelade T., Alqahtani j., and Canciani G., 2020, Prognosis of COVID-19 in Patients with Liver and Kidney Diseases: An Early Systematic Review and Meta-Analysis, Trop. Med. Infect. Dis., 5 (80): 1 14. doi:10.3390/tropicalmed5020080.

[36] Bonita R., Beaglehole R., and Kjellström T., 2006, Basic epidemiology $2^{\text {nd }}$ edition. World Health Organization 2006. Date of access: 16/10/2020. https://apps.who.int/iris/bitstream/handle/10665/43 541/9241547073_eng.pdf?sequence=1\&isAllowed $=\mathrm{y}$.

[37] Mancia G., Fagard R., Narkiewicz K., Redon J., Zanchetti A., Böhm M., et al., 2013, 2013 ESH/ESC guidelines for the management of arterial hypertension: The Task Force for the Management of Arterial Hypertension of the European Society of Hypertension (ESH) and of the European Society of Cardiology (ESC). Eur Heart J., 34(28): 2159-219. DOI: 10.1093/eurheartj/eht151.

[38] Cosentino F., Grant P.J., Aboyans V., Bailey
C.J., Ceriello1 A., Delgado V., et al., 2019, 2019 European Society of Cardiology (ESC) Guidelines on diabetes, pre-diabetes, and cardiovascular diseases developed in collaboration with the European Association of the Study of Diabetes (EASD). European Heart Journal, 00: 1 - 69. DOI:10.1093/eurheartj/ehz486.

[39] World Health Organization, 2009, A Training Manual for Health Workers on Healthy Lifestyle: An Approach for the Prevention and Control of NonCommunicable Diseases. Date of access: 1/10/2020. http://www.wpro.who.int/philippines/publications/t rainersguide.pdf.

[40] Kelly J, \& Shull J., 2019, Foundations of Lifestyle Medicine: The Lifestyle Medicine Board Review Manual. $2^{\text {nd }}$ ed. (Chesterfield, MO: United States of America).

[41] United Nations, 2011, Political declaration of the High-level Meeting of the General Assembly on the Prevention and Control of Non-communicable Diseases. United Nations 2011, Date of access: 15/10/2020.

https://www.who.int/nmh/events/un_ncd_summit2 011/political_declaration_en.pdf?ua=1.

[42]Hunter C.L., Rubin H., and Kim K., 2020, COVID-19 Economic Impacts: Beware the Ides of March - A Day Romans Settled Debts, KPMG Economics 2020, Date of access: 15/10/2020. https://assets.kpmg/content/dam/kpmg/cl/pdf/202003-kpmg-chile-advisory-coronavirus-mapping.pdf. [43] Alschuler L, Weil A, Horwitz R, Stamets P., Chiassona A., Crockera R., and Maizesa V., 2020, Integrative considerations during the COVID-19 pandemic, Explore, (00): 1 - 3 .

[44] World Health Organization, 2007, Prevention of Cardiovascular Disease: Guidelines for Assessment and Management of Cardiovascular Risk. Date of access: 8/10/2020. http://www.who.int/cardiovascular_diseases/guideli nes/Full\%20text.pdf.

[45] Brunzell, J. D., Davidson, M., Furberg, C. D., Goldberg, R. B., Howard, B. V., Stein J. H., et al., 2008, Lipoprotein Management in Patients with Cardiometabolic Risk: Consensus Conference Report from the American Diabetes Association and the American College of Cardiology Foundation, $J$. Am. Coll. Cardiol., 51, 1512 - 1524. DOI: 10.1016/j.jacc.2008.02.034.

[46] University of Leicester, 2012, Updated. The Handbook for Vascular Risk Assessment, Risk Reduction and Risk Management. Date of access: 31/12/2016. https://legacyscreening.phe.org.uk/policydb_download.p hp?doc $=259$. 